\title{
Novel chromosomal abberation in a patient with a unique sleep disorder
}

\author{
Yoshiya Hasegawa, Manabu Morishita, Akio Suzumura
}

\begin{abstract}
A 45 year old woman presenting with periodic hypersomnia for 17 years is reported on. She would sleep for three weeks followed by the same period awake. Polysomnography in the somnolent period disclosed an excess of total sleeping time with remarkably increased stage $1,3 / 4$, and REM sleep, without cataplexy or sleep paralysis. HLA typing was incompatible with narcolepsy or REM sleep behavioural disorder. Her chromosomes showed premature centromere division with chromatid puffing in areas of constitutive heterochromatin, which is exclusively found in the syndrome of infants termed Roberts' syndrome/SC phocomelia. Other laboratory findings were not normal. It is suggested that the present case is a novel sleep disorder related to a unique chromosomal aberration.
\end{abstract}

(F Neurol Neurosurg Psychiatry 1998;64:113-116)

Premature centromere division with chromatid puffing is a rare chromosomal aberration which is exclusively found in Roberts' syndrome. ${ }^{1}$ This is a report of the first adult case with the above chromosome aberration presenting a unique sleep disorder as the main manifestation, but without malformations seen in Roberts' syndrome. Polysomnography disclosed an excess of total sleeping time with $43 \%$ of REM sleep, which differs from the patterns of any known hypersomnia such as narcolepsy or periodic hypersomnia. ${ }^{2}$

Neurology, Prefectural Tajimi Hospital, 5-161 Maehata, Tajimi 507

Japan

Y Hasegawa

M Morishita

A Suzumura

Correspondence to: Dr Yoshiya Hasegawa, Department of Neurology, Nagoyacity Koseiin Geriatric Hospital, 2-1501 Sekobo

Meito-ku, Nagoya 465 Japan.

Telephone 008152704

2211; fax 0081527042780 .

Received 15 October 1996 and in final revised form 24 June 1997

Accepted 30 June 1997

\section{Case history}

A 45 year old woman was admitted to our hospital for the evaluation of her episodes of periodic hypersomnia which began at the age of 28 . She was born mildly asphyxiated and weighed $1700 \mathrm{~g}$ at 36 weeks of gestation. Her physical and mental development were slow; she first walked at the age of 3 . She had graduated from a special junior high school for physically and mentally disabled children, and had worked in a sewing factory for 13 years. She had never been married or pregnant. Her menarche was at 16 and menopause was at 43 years of age. She had a cerebellar haemorrhage when she was 37 years old. Bilateral visual disturbance due to optic nerve atrophy developed after 40 years of age. Her parents were first cousins. There was no family history of neurological or psychiatric disorders.

When she was 28 years old, she began to fall asleep for two to five days without any prodrome. After finishing the somnolent period, she could work in the factory at the beginning of the illness. However, the duration of the hypersomnia became longer within two years. She slept for almost three weeks and this was followed by the same duration of wakefulness. During the somnolent period, she slept continuously unless her mother woke her to eat light meals and for urination. When she awoke in remission, she ate much and stayed awake until midnight and this was followed by three to five hours of listlessness to a drowsy state. The

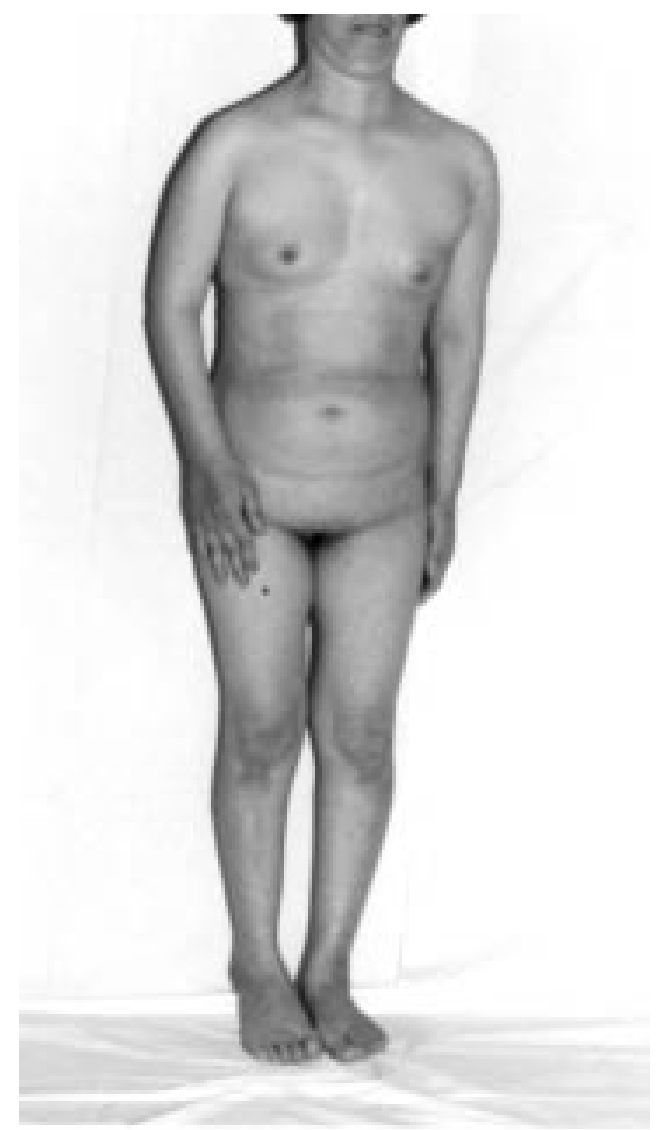

Figure 1 Patient at 45 years of age. She had no obvious physical anomalies of her body or limbs. 

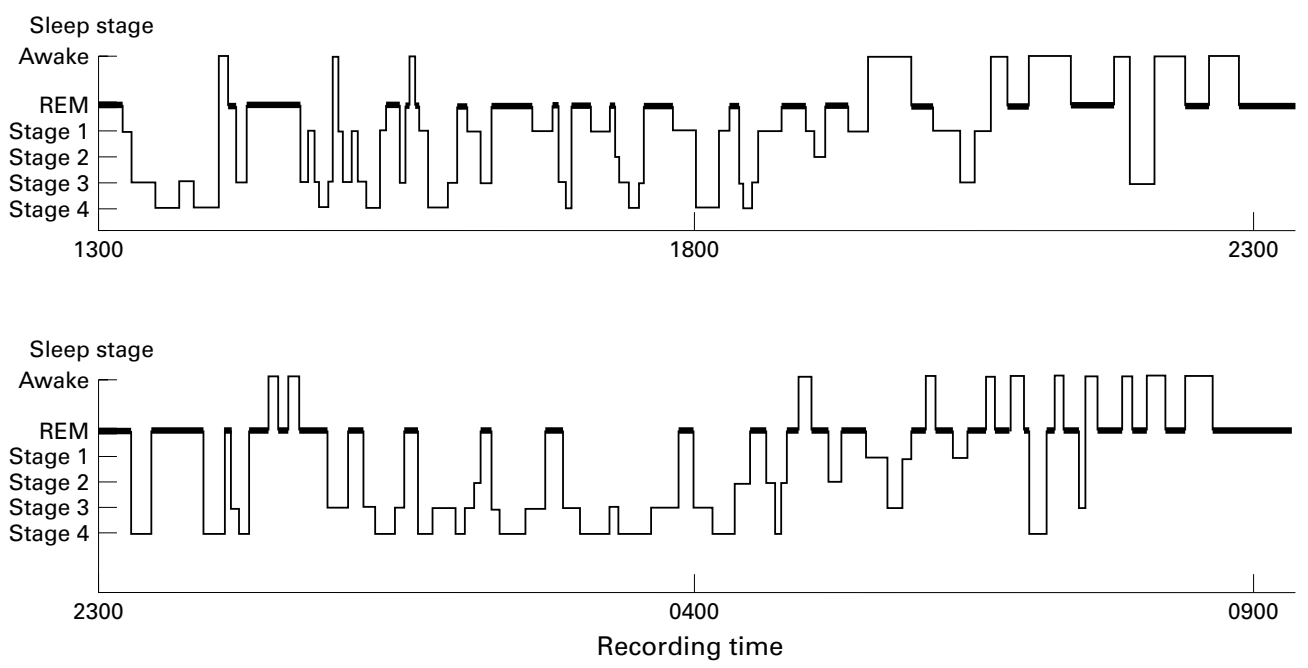

Figure 2 Sleep profile of polysomnography in somnolent stages. Total sleeping time was 924 minutes in a total 1200 minutes recording time. Total sleeping time comprised $16.3 \%$ of stage $1,14.6 \%$ of stage $2,26.1 \%$ of stage $3-4$, and $43 \%$ of REM sleep. The percentage of stage 1, stage 3/4, and REM sleep in total sleeping time were also significantly increased. The non-REM and REM sleep cycles were irregular and REM stage appeared at random.

remission periods also lasted three weeks and then another somnolent period would begin. The cycle continues until now, even after admission. Menstruation did not affect this cycle. She had not received any medication.

On admission, she was a thin, short women; $138 \mathrm{~cm}$ in height and $29 \mathrm{~kg}$ in weight. She had a relatively small face and head with a maximum head circumference of $47 \mathrm{~cm}$ and looked older than her actual age. She had no obvious physical anomalies of her body or limbs except for a mild high arched palate. The physical examination was not informative, including secondary sex characteristics (fig 1). Although her responses were slightly slow, she was alert and cooperative. Her intelligence score was 67 on the Wechsler adult intelligence scale.

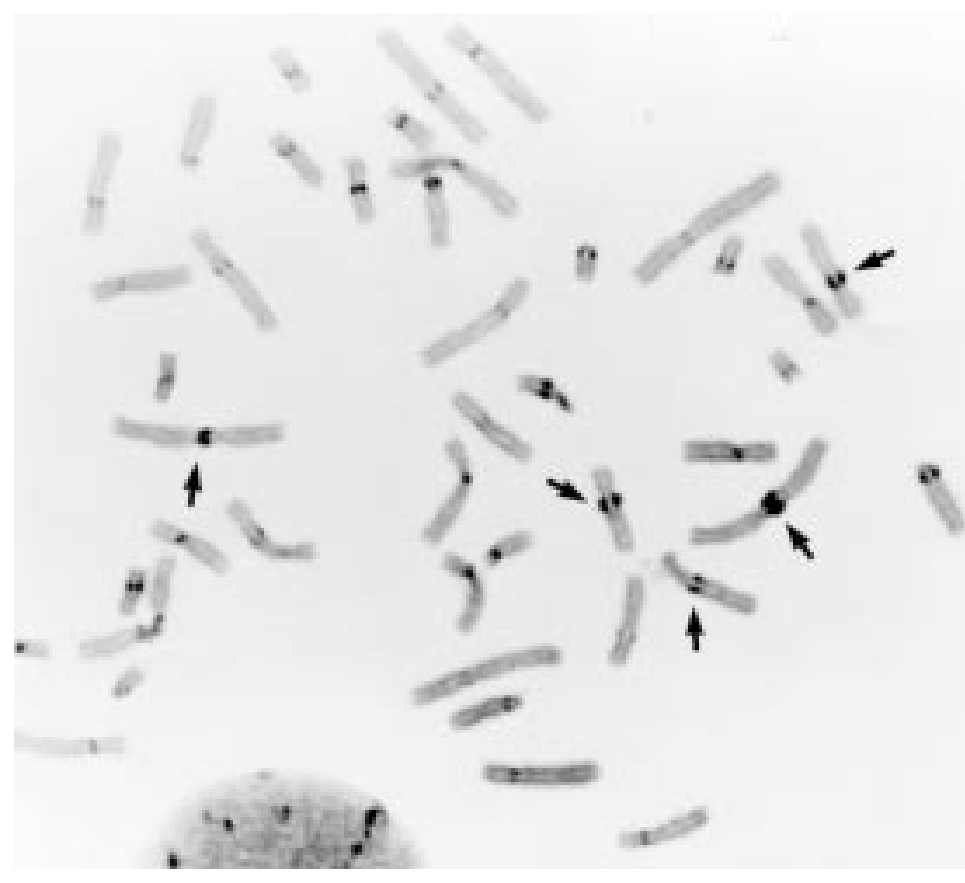

Figure $3 \quad C$ banded metaphase from the patient. All the chromosomes show the typical Roberts' syndrome effect, with centromere division and chromatid puffing (repulsion) in areas of constitutive heterochromatin (arrows indicate typical ones).
Neurological examination disclosed bilateral optic nerve atrophy and mild bilateral facial palsy of central type. Her visual acuity was $5 / 20$ in the left eye and she was almost blind in the right eye. The remaining cranial nerves were intact. Muscle tone, strength, and tendon reflexes were normal and planter reflexes were flexor. There was no evidence of cataplexy or sleep paralysis. Other neurological examinations, including tests for cerebellar function, were also normal.

Urinalysis, routine blood tests, ECG, and chest radiography were normal. Endocrinological studies showed normal urinary 17ketosteroid and 17-hydroxycorticosteroid concentrations and normal blood growth hormone, thyroid stimulating hormone, follicle stimulating hormone, prolactin, aldosterone, and adrenocorticotrophic hormone in the somnolent and alert periods. Her CSF was acellular and the content of protein, sugar, homovanillic acid, vanillylmandelic acid, 5-hydroxyindolacetic acid, serotonin, and $\gamma$-aminobutyric acid concentrations were normal in both the somnolent and alert periods. Serum and CSF concentrations of amino acids were normal. HLA typing of DQB1 was 0601/ 0601 and DR2 was positive. Cranial CAT/CT disclosed periventricular lucencies in the vicinity of the anterior horns of the lateral ventricle and slight cerebral atrophy. Brain MRI showed high signal intensity areas in the bilateral thalamus, putamen, and white matter in $\mathrm{T} 2$ weighed images and low signal intensity in the corpus callosum in T 1 weighed images. Single photon emission CT (SPECT) was considered to be normal in both somnolent and alert periods. EEG in the somnolent period showed a slower and more irregular basic rhythm than that in the alert period, which was accompanied by occasional slow waves of moderate amplitude.

The pattern of her sleep was analysed in the somnolent period by polysomnography, using an ambulatory EEG monitoring system and standard techniques. ${ }^{3}$ Total sleeping time was 
924 minutes in a total 1200 minutes. recording time. Total sleeping time was composed of $16.3 \%$ of stage $1,14.6 \%$ of stage $2,26.1 \%$ of stages $3-4$, and $43 \%$ of REM sleep. Non-REM and REM sleep cycles were irregular and the REM stage appeared at random (fig 2).

Cytogenetic analysis was performed on her peripheral blood lymphocytes cultured with phytohaemagglutinin. We analysed 50 cells which showed 10 cells of $45 \mathrm{X}$ and 40 cells of 46XX. Metaphase chromosomes showed a mosaicism of $45 \mathrm{X} / 46 \mathrm{XX}$ and showed premature centromere division and chromatid puffing which were designated as a "typical Roberts' syndrome effect"1 in all areas of constitutive heterochromatin (fig 3).

\section{Discussion}

Periodic hypersomnia, first reported by Kleine in 1925, is a syndrome characterised by hypersomnia persisting for two days to three weeks. ${ }^{4}$ Levin reported a syndrome associated with morbid hunger in the hypersomnia stage. ${ }^{5}$ The syndrome is now called Kleine-Levin syndrome, and classified as a specific type of periodic hypersomnia. ${ }^{6}$ As periodic hypersomnia rarely shows strict periodicity, it is now called recurrent hypersomnia (the International Classification of Sleep Disorders issued in 1990). ${ }^{7}$ The hypersomnia seen in the present case is unique. The exact alternative cycle of hypersomnia and wakefulness is different from that seen in periodic hypersomnia. The long lasting course in this patient is also different from the course of periodic hypersomnia or intermittent repetitive pseudocoma reported by Lugaresi et al. ${ }^{8}$ Clinical features and HLA typing are not compatible with narcolepsy ${ }^{9}$ and the REM sleep behaviour disorder. ${ }^{10}$ Analysis with polysomnography showed another unique point of her hypersomnia. Total sleeping time of normal subjects was composed of $5-10 \%$ of stage $1,50 \%$ of stage $2,20 \%$ of stage $3-4$, and $20 \%-25 \%$ of REM sleep. Sleep pattern of periodic hypersomnia was reported as follows ${ }^{11}{ }^{12}$; total sleeping time and the percentage of REM sleep are identical to normal controls, whereas the percentage of stage 2 is decreased and that of stage $3 / 4$ is increased. Her actual sleep time was $77 \%$ of total recording time, which was obviously prolonged compared with periodic hypersomnia and healthy subjects. The percentage of stage 1 , stage $3 / 4$, and REM sleep in total sleeping time were also remarkably increased compared with periodic hypersomnia. The REM-non-REM sleeping cycle was irregular. This indicates that her sleep was different from those of either normal subjects or the patients with periodic hypersomnia and was characterised by a pattern of increased deep sleep and REM sleep, and decreased light sleep. Although we could not detect any anatomical or chemical abnormalities which induced the sleep cycle, some mediators such as cytokines or other soluble factors may regulate these unusual sleep-remission cycles and REM-non-REM cycles. It has been shown recently that interleukin-1, tumour necrosis factor (TNF), or hormones such as growth hormone releasing hormone induce sleep and regulate the cycle of REM and non-REM sleep. ${ }^{13-14}$ (TNF $\alpha$ was undetectable in her CSF).

Her chromosomes displayed very rare abnormalities reported exclusively in Roberts' syndrome. Petrinelli et al reported similar chromosomal abnormalities in the sibling of Roberts' syndrome, who had no physical or neurological abnormality. ${ }^{15}$ However, sleep disorder accompanied by the similar chromosomal aberration has not been reported. Roberts' syndrome is a rare autosomal recessive condition characterised by pronounced tetraphocomelia, midfacial clefts, severe mental and physical retardation, and death at a very early age, none of which was found in the present case. ${ }^{16}$ Although her chromosomes showed a mosaicism in $45 \mathrm{X} / 46 \mathrm{XX}$, she did not have characteristics of Turner's syndrome except for short stature and late menarche.

It remains to be elucidated whether or not the chromosomal aberration was causally related to the sleep disorder. Because she had cerebellar haemorrhage and MRI disclosed abnormal lesions in the thalami, it is possible that the sleep disorder may be secondary to the vascular lesions. It is also unknown whether the chromosomal aberration induces vascular abnormalities. Bassetti et al reported hypersomnia after a paramedian thalamic stroke. However, paramedian thalamic stroke has an acute onset and has not been reported to show periodic hypersomnia. Polysomnography in paramedian thalamic stroke has shown an increased stage 1 and normal REM sleep. ${ }^{17}$ Thus we suggest that the present case is a novel sleep disorder related to a unique chromosome aberration.

We thank Dr Donald H Silberberg, Professor of Neurology, University of Pennsylvania, and Richard F Mayer, Professor of Neurology, University of Maryland for commenting on the manuscript.

1 German J. Roberts' syndrome. I. Cytological evidence for a disturbance in chromatid pairing. Clin Genet 1979;16:4417 .

2 Aldrich MS. The clinical spectrum of narcolepsy and idiopathic hypersomnia. Neurology 1996;46:393-401.

3 Rechtschafffen A, Kales A. A manual of standardized terminology, techniques, and scoring system for sleep stages of human subjects. Los Angeles: Brain Information Service, Brain Research Institute, 1968.

4 Kleine W. Periodsche Schlafsucht. Mschr Psychiatric Neurologice 1925;57:285-320.

5 Levin M. Periodic somnolence and morbid hunger; a new syndrome. Brain 1936;59:494-504.

6 Critchley M, Hoffman HL. The syndrome of periodic somnolence and morbid hunger (Kleine-Levin syndrome). BMF 1942;138:4230-2.

7 Thorpy MJ, and the Diagnostic Classification Steering Thorpy $M J$, and the Diagnostic Classification Steering
Committee. The international classification of sleep disorders: diagnostic and coding manual. Rochester, Minnesota: diagnostic and coding manual. Rochester,

8 Lugaresi E, Medori R, Montagna P, et al. Fatal familiar insomnia and dysautonomia with selective degeneration of thalamic nuclei. N Engl F Med 1986;315:997-1003.

9 Matsuki K, Grumet FC, Xiaoyan L, et al. DQ (rather than DR) gene marks susceptibility to narcolepsy. Lancet 1992;339:1052-4.

10 Schenck CH, Garcia-Rill E, Segall M, et al. HLA class genes associated with REM sleep behavior disorder. Ann Neurol 1996;39:261-3.

11 Barontini F, Zappoli R. A case of Kleine-Levin syndromeclinical and polygraphic study. In: Gastant $\mathrm{H}$, et al, eds. The Abnormalities of Sleep in Man. Bologna, 1968;238-46.

12 Lavie P. Sleep patterns in Kleine-Levin syndrome. Electroencephalogr Clin Neurophysiol 1979;47:369-71.

13 Krueger JM, Obal JF. GHRH and IL-1 in sleep regulation. FASEB $\mathcal{F}$ 1993;7:645-52. 
14 Obal JF, Fang J, Payne LC, et al. Growth hormone-releasing hormone (GHRH) mediates the sleep promoting activity of hormone (GHRH) mediates the sleep promoting activity of interleukin-

15 Petrinelli P, Antonelli A, Marucci L, et al. Premature centromere splitting in a presumptive mild form of Roberts syndrome. Hum Genet 1984;66:96-9.
16 Van Den Berg DJ, Francke U. Roberts syndrome; a review of 100 cases and a new rating system for severity. $A m \mathcal{F} M e d$ Genet 1993;47:1104-23.

17 Bassetti C, Mathis J, Gugger M, et al. Hypersomnia following paramedian thalamic stroke: a report of 12 patients. Ann Neurol 1996;39:471-80. 